\title{
Clinical outcomes of non-alcoholic fatty liver disease: Polish-case control study
}

\author{
Radosław Kempiński $i^{1, A-F}$, Agata Łukawska ${ }^{2, B, D-F}$, Filip Krzyżanowski ${ }^{2, D-F}$, \\ Dominika Ślósarz ${ }^{2, B, D-F}$, Elżbieta Poniewierka ${ }^{1, A, D-F}$ \\ ${ }^{1}$ Department of Gastroenterology and Hepatology, Wroclaw Medical University, Poland \\ ${ }^{2}$ Gastroenterology Student Organization, Wroclaw Medical University, Poland \\ A - research concept and design; $B$ - collection and/or assembly of data; $C$ - data analysis and interpretation; \\ $\mathrm{D}$ - writing the article; $\mathrm{E}$ - critical revision of the article; $\mathrm{F}$ - final approval of the article
}

Address for correspondence

Radosław Kempiński

E-mail: radoslaw.kempinski@umed.wroc.pl

Funding sources

None declared

Conflict of interest

None declared

Received on February 28, 2019

Reviewed on March 15, 2019

Accepted on April 4, 2019

Published online on April 23, 2019

Cite as

Kempiński R, Łukawska A, Krzyżanowski F, Ślósarz D,

Poniewierka E. Clinical outcomes of non-alcoholic fatty liver disease: Polish-case control study. Adv Clin Exp Med. 2019;28(12):1615-1620. doi:10.17219/acem/106173

DOI

10.17219/acem/106173

\section{Copyright}

Copyright by Author(s)

This is an article distributed under the terms of the

Creative Commons Attribution Non-Commercial License

(http://creativecommons.org/licenses/by-nc-nd/4.0/)

\begin{abstract}
Background. Non-alcoholic fatty liver disease (NAFLD) is becoming the most common cause of chronic liver disease worldwide, affecting up to $30 \%$ of population. Non-alcoholic fatty liver disease can lead to non-alcoholic steatohepatitis (NASH), fibrosis, cirrhosis, and hepatocellular carcinoma. Age, obesity, insulin resistance, type 2 diabetes, and dyslipidemia are important risk factors for developing hepatic steatosis. Concomitant diseases, especially cardiovascular, are discussed as important causes of death in NAFLD patients.
\end{abstract}

Objectives. The objective of this study was to conduct a retrospective comparison of the frequency of concomitant diseases in NAFLD patients and controls, especially metabolic syndrome and cardiovascular disease (CVD).

Material and methods. A total of 1,058 (558 NAFLD patients and 500 controls). Diagnosis of NAFLD was established with ultrasound examination in the absence of other causes of fatty liver. The control group included patients with no history of liver disease, normal liver image in ultrasound examination and normal liver laboratory tests.

Results. Overweight and/or obesity were diagnosed in $80.8 \%$ of patients in the study group and $40.8 \%$ in the controls $(p<0.001)$. Metabolic syndrome was present in $48.7 \%$ patients in the study group compared with $14.4 \%$ controls, $(p<0.001)$. In the study group, we found higher prevalence of hypertension $(56.1 \%$ vs 37\%; $p<0.001$ ), type 2 diabetes mellitus (24.4\% vs $8.6 \% ; p<0.001$ ), decreased concentration of serum HDL (35.1\% vs 19.5\%; $p<0.001$ ), elevated serum triglycerides (36.5\% vs $15.4 \% ; p<0.001)$. Cardiovascular disease was found in $13.6 \%$ of individuals in the study group and in $15 \%$ controls (NS, $p=0.32$ ). The most frequent concomitant gastrointestinal disease present in the study group was gastroesophageal reflux disease (GERD) (31.9\% vs 22.8\%; $p<0.001$ ) followed by colonic diverticulosis (23.7\% vs 15.8\%; $p<0.005)$.

Conclusions. Metabolic syndrome with its components is more common in NAFLD patients compared to matched controls. Additionally, NAFLD patients are more often affected by GERD and colonic diverticulosis but not by CVD.

Key words: metabolic syndrome, cardiovascular disease, concomitant diseases, non-alcoholic fatty liver disease 


\section{Introduction}

Non-alcoholic fatty liver disease (NAFLD) recently has become the predominant cause of chronic liver disease in many parts of the world. ${ }^{1}$ The spectrum of the disease ranges from simple liver steatosis through non-alcoholic steatohepatitis (NASH) with possible fibrosis leading to cirrhosis. Furthermore, hepatocellular carcinoma is the complication of liver cirrhosis in patients with NAFLD. In the last decade, NAFLD was revealed to be multisystem disease, affecting also extra-hepatic organs. ${ }^{2}$ Non-alcoholic fatty liver disease is diagnosed in approx. 30-40\% of men and $15-20 \%$ of women. ${ }^{3}$ In certain subgroups, like type 2 diabetes mellitus (T2DM) individuals, it occurs even in up to $70 \%$ of this group of patients. ${ }^{4}$ Increasing incidence rates of NAFLD are related to the growing prevalence of obesity that is associated with a wide range of complications, including metabolic syndrome (MetS). Non-alcoholic fatty liver disease is strongly connected with metabolic syndrome and its components. Many cross-sectional studies have demonstrated that NAFLD is significantly associated with MetS. ${ }^{5-8}$ Recent data showed that NAFLD increased the overall mortality by $57 \%$, mainly from liver-related and cardiovascular disease (CVD) causes, and the risk of T2DM was increased approx. twofold. ${ }^{9}$ Cardiovascular disease is the most common cause of death globally, with an estimated 17.9 million people dying from CVD in 2016. Emerging evidence shows that NAFLD is also connected to other chronic diseases, such as endocrinopathies (e.g., hypothyroidism, polycystic ovary syndrome, hypogonadism), colorectal cancer, sleep apnea, osteoporosis, and psoriasis. ${ }^{10}$ Recent meta-analysis reported that NAFLD was associated with a twofold increased risk of chronic kidney disease. ${ }^{11}$ As colonic diverticulosis shares the same pathways with NAFLD (obesity, hypertension, dyslipidemia) ${ }^{12}$ and its risk is higher in patients with visceral fat accumulation. ${ }^{13}$ The possible connection between NAFLD and colonic diverticulosis is additionally investigated.

Liver biopsy is still a gold standard for the diagnosis of NAFLD and its stage (simple steatosis, steatohepatitis, fibrosis, cirrhosis). It is an invasive procedure, requiring most often hospital admission. Therefore, non-invasive methods were recently implemented, especially to identify the patients with liver fibrosis. Abdominal ultrasound is a simple method to diagnose liver steatosis, but, unfortunately, like computed tomography or magnetic resonance techniques, it is not accurate in defining fibrosis in the liver. Transient elastography estimates liver tissue stiffness in ultrasound method and is a novel imaging technique to evaluate patients with liver fibrosis. Serum cytokeratin-18 is a promising and accurate noninvasive marker of non-alcoholic steatohepatitis (NASH). The staging of liver fibrosis with simple serum marker panels is still a challenge, the most accurate are FIB-4 test and NAFLD fibrosis score. ${ }^{14}$ A novel method of fibrosis detection in NAFLD is ${ }^{13} \mathrm{C}$-methacetin breath test. ${ }^{15}$
The objective of our study was a retrospective evaluation of patients with NAFLD and a comparison of the frequency of concomitant diseases in NAFLD patients and controls, especially metabolic syndrome with its components and CVD.

\section{Material and methods}

\section{Study group}

A total of 2,309 consecutive hospital charts of patient hospitalized in the Department and Clinic of Gastroenterology and Hepatology of Wroclaw Medical University between 2017-2018 were meticulously searched. Four hundred twenty-two individuals were excluded due to secondary causes of liver injury: viral hepatitis, autoimmune hepatitis, hemochromatosis, Wilson's disease, $\alpha-1$ antitrypsin deficiency, drug-induced hepatic injury, cholestatic liver disease, and alcohol consumption higher than $30 \mathrm{mg} /$ day for men and $20 \mathrm{mg} /$ day for women.

One thousand eighty-seven patients were left for analysis. The study NAFLD group consisted of 558 consecutive patients with liver steatosis. Five hundred individuals with no history of liver disease, normal liver laboratory tests and normal image of liver on ultrasound examination were matched for age and sex with the study group and served as controls. Flowchart for patient inclusion was shown in Fig. 1.

Diagnosis of type 2 diabetes mellitus (T2DM) was defined as a registered diagnosis in patient charts, a nonfasting glucose value $\geq 180 \mathrm{mg} / \mathrm{dL}$ or a fasting glucose value $\geq 126 \mathrm{mg} / \mathrm{dL}$, or having treatment for diabetes. Hypertension was diagnosed when there was a registered diagnosis in the patient's chart, a resting blood pressure $\geq 140 / 90 \mathrm{~mm} \mathrm{Hg}$ or if the patient had any anti-hypertensive drug prescription. Dyslipidemia was defined

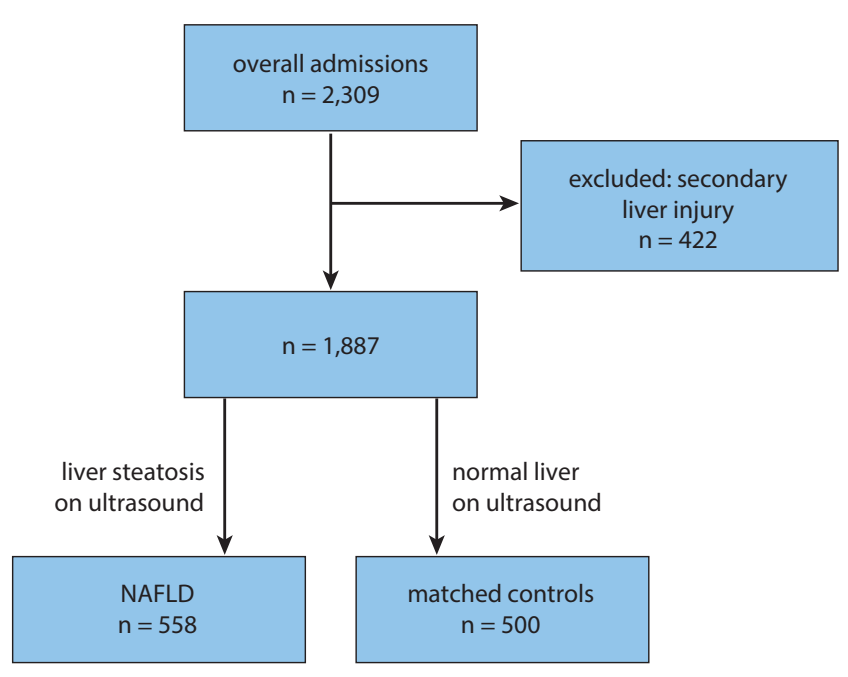

Fig. 1. Flowchart for patient inclusion 
when the patient had a fasting triglyceride concentration value $\geq 150 \mathrm{mg} / \mathrm{dL}$ or $\mathrm{HDL}$-cholesterol value $<40 \mathrm{mg} / \mathrm{dL}$ (male), $<50 \mathrm{mg} / \mathrm{dL}$ (female). Body mass index (BMI) was calculated as (weight $[\mathrm{kg}] /$ height $[\mathrm{m}]^{2}$ ). The diagnosis of metabolic syndrome was established according to Adult Treatment Panel III criteria. ${ }^{16}$ Cardiovascular disease was defined as one of the following: coronary heart disease, cerebrovascular disease, peripheral arterial disease, rheumatic heart disease, congenital heart disease, deep vein thrombosis, or pulmonary embolism. The diagnosis of colonic diverticulosis was based on an endoscopy and/ or radiological examination.

Laboratory parameters extracted from patients' charts were blood morphology, AST, ALT, HDL-cholesterol, triglycerides, and glucose. Other parameters were incomplete and not included in the study.

The diagnosis of liver steatosis was defined in ultrasound examination. Patients were examined in the supine and left lateral decubitus position under fasting conditions. A gastroenterologist with over 10 years of abdominal ultrasound experience evaluated the echogenicity of the liver and the right kidney. Increased hepatorenal echogenicity, bright hepatic echoes, and vascular blurring of portal or hepatic vein were classified as exclusive features of NAFLD.

\section{Statistical analysis}

Continuous, normally distributed variables were summarized as mean \pm standard deviation (SD). Student's t-test was performed to compare the means in groups with normally distributed data. In groups with non-normal distribution, the Mann-Whitney U-test was used. To compare mean prevalence differences between groups, the $X^{2}$ test (categorical variables) was performed. Statistical analysis was performed using STATISTICA v. 13.3 software (StatSoft Inc., Tulsa, USA).

\section{Ethical considerations}

The study protocol was approved by local ethics committee in accordance with the Helsinki Declaration.

\section{Results}

Five hundred fifty-eight consecutive patients with liver steatosis and 500 controls were enrolled into the study. According to the absence of other causes of liver steatosis, all the patients in the study group were classified as NAFLD. Twenty-four patients (4.3\%) in this group were diagnosed with liver cirrhosis. The mean age of the patients was 58.1 years in NAFLD group and 57.5 years in the controls, respectively. Individuals in the $7^{\text {th }}$ decade of life were most often represented. A total of $50.4 \%$ of the patients in the study group were male. The mean age and the sex distribution in both groups did not differ statistically significantly. We tried to estimate the prevalence of NAFLD in all the patients admitted to the gastroenterology department: 558 cases out of 2,309 overall admissions $=24.2 \%$. Selected clinical data of patients in NAFLD patients and controls is shown in Table 1.

The mean BMI was significantly higher in the study group compared to controls (29.2 vs 24.4, p < 0.001). Patients with NAFLD had significantly higher serum TG and lower HDL concentration.

The prevalence of metabolic syndrome and its components in NAFLD patients and controls were estimated (Table 2, number of patients in the brackets in cases where the missing data made it impossible to complete the calculation). Not surprisingly, MetS with components (hypertension, T2DM, dyslipidemia) was strongly correlated with NAFLD. The concomitant diseases in NAFLD and controls were evaluated. We managed to estimate the prevalence of consecutive diseases with odd ratios (Table 3). The concomitant diseases connected with NAFLD were: overweight/obesity, gastroesophageal reflux disease (GERD), colonic diverticulosis and cholecystolithiasis. Functional gastrointestinal diseases were less common in NAFLD than in the controls. The prevalence of other diseases was not significantly different in the study group and controls.

We chose FIB-4 scoring system to estimate the possibility of coexisting fibrosis. ${ }^{17}$ In 348 patients (62.4\%), the FIB-4 score was lower than 1.45 , suggesting no advanced fibrosis present. These patients will not require

Table 1. Selected clinical data of NAFLD patients and controls

\begin{tabular}{|c|c|c|c|}
\hline Clinical data & NAFLD & Controls & $\mathrm{p}$-value \\
\hline Age [years] ${ }^{a}$ & $58.1 \pm 14.1$ & $57.5 \pm 16.2$ & 0.73 \\
\hline Sex (female/male) & $277 / 281$ & $252 / 248$ & 0.81 \\
\hline BMI $\left[\mathrm{kg} / \mathrm{m}^{2}\right]^{\mathrm{a}}$ & $29.2 \pm 5.5$ & $24.4 \pm 3.9$ & $<0.001$ \\
\hline $\mathrm{ALT}[\mathrm{U} / \mathrm{L}]^{\mathrm{a}}$ & $33.5 \pm 32.5$ & $26.2 \pm 16.5$ & $<0.001$ \\
\hline AST [U/L $]^{\mathrm{a}}$ & $31.8 \pm 24.0$ & $22.5 \pm 16.8$ & $<0.001$ \\
\hline Glucose $[\mathrm{mg} / \mathrm{dL}]^{\mathrm{a}}$ & $108.5 \pm 30.6$ & $103.6 \pm 32.2$ & 0.35 \\
\hline $\mathrm{TG}[\mathrm{mg} / \mathrm{dL}]^{\mathrm{a}}$ & $147.1 \pm 86.8$ & $108.4 \pm 60.5$ & $<0.001$ \\
\hline HDL (male: $>40$ mg/dL, female: $>50$ mg/dL) ${ }^{a}$ & $50.1 \pm 14.4$ & $56.6 \pm 16.7$ & $<0.001$ \\
\hline
\end{tabular}

ALT - alanine transaminase; AST - aspartate transaminase; HDL - high-density lipoprotein; BMI - body mass index; TG - triglycerides; ${ }^{\text {a }}$ mean, standard deviation (SD). 
Table 2. The prevalence of metabolic syndrome and its components in NAFLD patients and controls

\begin{tabular}{|c|c|c|c|c|c|}
\hline Components of metabolic syndrome & NAFLD & {$[\%]$} & Controls & {$[\%]$} & p-value \\
\hline Metabolic syndrome & $268(n=550)$ & 48.7 & $72(n=487)$ & 14.4 & $<0.001$ \\
\hline Hypertension & 313 & 56.1 & 185 & 37 & $<0.001$ \\
\hline Type 2 diabetes mellitus & 136 & 24.4 & 43 & 8.6 & $<0.001$ \\
\hline Hyperlipidemia (hypertriglyceridemia) & $195(n=534)$ & 36.5 & $75(n=487)$ & 15.4 & $<0.001$ \\
\hline Hyperlipidemia (decreased HDL) & $183(n=522)$ & 35.1 & $91(n=467)$ & 19.5 & $<0.001$ \\
\hline
\end{tabular}

NAFLD - non-alcoholic fatty liver disease; HDL - high-density lipoprotein

Table 3. Concomitant diseases in NAFLD patients and controls

\begin{tabular}{|c|c|c|c|c|c|c|}
\hline Concomitant diseases & $\begin{array}{l}\text { NAFLD } \\
(\mathrm{n}=558)\end{array}$ & {$[\%]$} & $\begin{array}{l}\text { Controls } \\
(n=500)\end{array}$ & {$[\%]$} & p-value & Odds ratio \\
\hline Overweight/obesity & 451 & 80.8 & 204 & 40.8 & $<0.0001$ & 6.12 \\
\hline GERD & 178 & 31.9 & 114 & 22.8 & 0.0009 & 1.59 \\
\hline Colonic diverticulosis & 132 & 23.7 & 79 & 15.8 & 0.0014 & 1.65 \\
\hline CVD & 76 & 13.6 & 75 & 15 & 0.32 & 0.89 \\
\hline Cholecystolithiasis & 74 & 13.3 & 36 & 7.2 & 0.0013 & 1.97 \\
\hline Hypothyroidism & 60 & 10.8 & 50 & 10 & 0.69 & 1.08 \\
\hline Crohn's disease & 60 & 10.8 & 38 & 7.6 & 0.078 & 1.46 \\
\hline Ulcerative colitis & 53 & 9.5 & 54 & 10.8 & 0.48 & 0.87 \\
\hline Cardiac dysrhythmia & 43 & 7.7 & 30 & 6.0 & 0.27 & 1.31 \\
\hline Pancreatitis & 43 & 7.7 & 32 & 6.6 & 0.41 & 1.22 \\
\hline Neoplasm & 38 & 6.8 & 37 & 7.4 & 0.71 & 0.91 \\
\hline Functional Gl diseases & 20 & 3.6 & 47 & 9.4 & 0.0001 & 0.36 \\
\hline Asthma & 17 & 3.0 & 14 & 2.8 & 0.81 & 1.09 \\
\hline Chronic kidney disease & 12 & 2.1 & 12 & 2.4 & 0.79 & 0.89 \\
\hline COPD & 11 & 2.0 & 10 & 2.0 & 0.97 & 0.99 \\
\hline
\end{tabular}

COPD - chronic obstructive pulmonary disease; CVD - cardiovascular disease; GERD - gastroesophageal reflux disease; GI - gastrointestinal.

further diagnostic procedure. Fifteen patients with FIB-4 score above 3.25 are likely to have advanced liver fibrosis. One hundred ninety-one (34.2\%) patients with intermediate score $1.45-3.25$ require additional diagnostic procedures (Fig. 2). These patients were sent for ambulatory transient elastography.

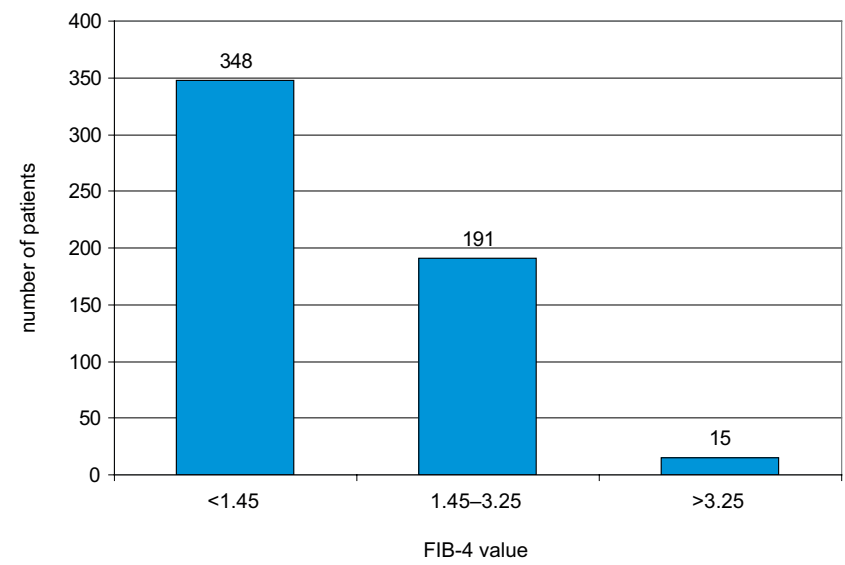

Fig. 2. FIB-4 score in patients with NAFLD

\section{Discussion}

Non-alcoholic fatty liver disease, a chronic condition of the liver related to hepatic steatosis, was recently recognized as the most common chronic liver disease. Increasing prevalence rates of the risk factors for NAFLD are the following: obesity, diabetes and metabolic syndrome will most probably result into further increasing incidence rates of NAFLD all over the world.

The mean age of the patients in the study group was 58.1. Non-alcoholic fatty liver disease was found slightly more often in men than in women (not statistically significant difference in our study group). This is consistent with the results of the epidemiological studies performed in the USA. The frequency of hepatic steatosis varies significantly with ethnicity (45\% in Hispanics; 33\% in Whites; $24 \%$ in Blacks). ${ }^{3}$ Our study group was monoethnical - 100\% individuals were White. We can only estimate the prevalence of hepatic steatosis in all the patients admitted to the gastroenterology department as approx. 25\% (558 out of 2,309).

Non-alcoholic fatty liver disease afflicts overweight and obese people and presents the coexistence with 
the MetS-associated disorders, like T2DM, hypertension and dyslipidemia. ${ }^{18}$ These comorbidities have a negative impact on the natural course of NAFLD. ${ }^{19}$ In fact, progression to fibrosis in NAFLD is highly influenced by the presence of T2DM and obesity. ${ }^{20,21}$ In a very recent review, authors have mapped shared gene/protein interaction networks and performed gene-disease analysis. ${ }^{18}$ Shared mechanisms among NAFLD and the MetS diseases were revealed and provided evidence that NAFLD and especially NASH, requires taking multi-target approaches, rather than focusing on single mechanisms of disease. Indeed, in our study we have confirmed strong connections between either MetS itself or its components and NAFLD. The prevalence of MetS was 48.7\%, overweight/ obesity was $80.8 \%$, atrial hypertension $56.1 \%$, T2DM $24.4 \%$, hypertriglyceridemia $36.5 \%$, and decreased HDL $35.1 \%$, respectively. The difference in all the cases was significantly different from the corresponding rates in the control group.

Unexpectedly, the frequency of CVD was almost the same in the study group compared to controls $(13.6 \%$ vs $15 \%)$. As patients with NAFLD have features of MetS, they also have important clinical implications for the development of future CVD. Many studies have addressed this issue before, finding the prevalence of CVD significantly higher in NAFLD patients. ${ }^{22}$ Recently, a vast European study has been performed in Sweden ${ }^{23}$ and its authors claim that it is the largest ever study of biopsyproven NAFLD. Over 600 patients were followed up during a mean of 20 years. The authors found that death rates due to cardiovascular reasons did not differ statistically in NAFLD group compared to controls. The most common gastrointestinal disease in NAFLD group was GERD. It was statistically more common compared to controls. Large cohort study (over 34,000 participants) showed that NAFLD is not independently associated with the risk of the development of reflux esophagitis after adjusting for BMI and other metabolic factors. ${ }^{24}$ The authors suggest that reflux esophagitis is primarily the consequence of increased BMI commonly associated with NAFLD. Probably for the same reason, cholecystolithiasis appeared more often in NAFLD patients, as both diseases share the same main risk factor: overweight and obesity. Moreover, this association is more strongly seen in females than in males. ${ }^{25}$

In the literature there is lacking evidence on connection between NAFLD and colonic diverticulosis. Only 1 casecontrol study claimed that diverticulosis in the elderly (>65 years) was a negative predictor of liver steatosis. ${ }^{26}$ Our findings in the younger group were different. In NAFLD patients, the prevalence of diverticulosis was much higher compared to controls.

We have evaluated the FIB-4 score that is simple and easy to calculate and to estimate the risk of fibrosis in the liver. In our study group, most cases were classified as not having fibrosis, whereas only $1 / 3$ of patients required additional examinations (for instance transient elastography).

\section{Conclusions}

Overweight and obesity as well as metabolic syndrome with its components: hypertension, type 2 diabetes mellitus, and dyslipidemia are more common in NAFLD patients compared to matched controls. No significant difference between the study group and controls was found in the frequency of CVD. Additionally, NAFLD patients are more often affected by GERD, colonic diverticulosis and cholecystolithiasis.

\section{ORCID iDs}

Radosław Kempiński (D) https://orcid.org/0000-0002-6030-2700 Agata Łukawska (D) https://orcid.org/0000-0002-3766-3073

Filip Krzyżanowski (D) https://orcid.org/0000-0002-7571-7925

Dominika Ślósarz (D) https://orcid.org/0000-0002-8159-1660

Elżbieta Poniewierka (D) https://orcid.org/0000-0002-2074-976X

\section{References}

1. Masuoka HC, Chalasani N. Nonalcoholic fatty liver disease: An emerging threat to obese and diabetic individuals. Ann N Y Acad Sci. 2013; 1281:106-122.

2. Armstrong MJ, Adams LA, Canbay A, Syn WK. Extrahepatic complications of nonalcoholic fatty liver disease. Hepatology. 2014;59(3): 1174-1197.

3. Browning JD, Szczepaniak LS, Dobbins R, Nuremberg P, Horton JD, Cohen JC. Prevalence of hepatic steatosis in an urban population in the United States: Impact of ethnicity. Hepatology. 2004;40(6):1387-1395.

4. Blachier M, Leleu H, Peck-Radosavljevic M, Valla DC, Roudot-Thoraval F. The burden of liver disease in Europe: A review of available epidemiological data. J Hepatol. 2013;58(3):593-608.

5. Hamaguchi M, Kojima T, Itoh Y, et al. The severity of ultrasonographic findings in nonalcoholic fatty liver disease reflects the metabolic syndrome and visceral fat accumulation. Am J Gastroenterol. 2007; 102(12):2708-2715.

6. Musso G, Gambino R, Bo S, et al. Should nonalcoholic fatty liver disease be included in the definition of metabolic syndrome? A crosssectional comparison with Adult Treatment Panel III criteria in nonobese nondiabetic subjects. Diabetes Care. 2008;31(3):562-568.

7. Kwon YM, Oh SW, Hwang SS, Lee C, Kwon H, Chung GE. Association of nonalcoholic fatty liver disease with components of metabolic syndrome according to body mass index in Korean adults. Am J Gastroenterol. 2012;107(12):1852-1858.

8. Speliotes EK, Massaro JM, Hoffmann U, et al. Fatty liver is associated with dyslipidemia and dysglycemia independent of visceral fat: The Framingham heart study. Hepatology. 2010;51(6):1979-1987.

9. Musso G, Gambino R, Cassader M, Pagano G. Meta-analysis: Natural history of non-alcoholic fatty liver disease (NAFLD) and diagnostic accuracy of noninvasive tests for liver disease severity. Ann Med. 2011;43(8):617-649.

10. Musso G, Cassader M, Olivetti C, Rosina F, Carbone G, Gambino R. Association of obstructive sleep apnoea with the presence and severity of non-alcoholic fatty liver disease. A systematic review and metaanalysis. Obes Rev. 2013;14(5):417-431.

11. Musso G, Gambino R, Tabibian JH, et al. Association of non-alcoholic fatty liver disease with chronic kidney disease: A systematic review and meta-analysis. PLoS Med. 2014;11(7):e1001680.

12. Kopylov U, Ben-Horin S, Lahat A, Segev S, Avidan B, Carter D. Obesity, metabolic syndrome and the risk of development of colonic diverticulosis. Digestion. 2012;86(3):201-205.

13. Nagata N, Sakamoto K, Arai T, et al. Visceral abdominal obesity measured by computed tomography is associated with increased risk of colonic diverticulosis. J Clin Gastroenterol. 2015;49(10):816-822.

14. Festi $D$, Schiumerini R, Marzi L, et al. Review article: The diagnosis of non-alcoholic fatty liver disease - availability and accuracy of noninvasive methods. Aliment Pharmacol Ther. 2013;37(4):392-400.

15. Kempiński R, Neubauer K, Wieczorek S, Dudkowiak R, Jasińska M, Poniewierka E. 13C-methacetin breath testing in patients with nonalcoholic fatty liver disease. Adv Clin Exp Med. 2016;25(1):77-81. 
16. Executive Summary of The Third Report of The National Cholesterol Education Program (NCEP) Expert Panel on Detection, Evaluation And Treatment of High Blood Cholesterol In Adults (Adult Treatment Panel III). JAMA. 2001;285(19):2486-2497.

17. Sterling RK, Lissen $\mathrm{E}$, Clumeck N, et al. Development of a simple noninvasive index to predict significant fibrosis in patients with HIV/HCV coinfection. Hepatology. 2006;43(6):1317-1325.

18. Sookoian S, Pirola CJ. Review article: Shared disease mechanisms between non-alcoholic fatty liver disease and metabolic syndrome - translating knowledge from systems biology to the bedside. Aliment Pharmacol Ther. 2019;49(5):516-527.

19. Rinella ME. Nonalcoholic fatty liver disease: A systematic review. JAMA. 2015;313(22):2263-2273.

20. Friedman SL, Neuschwander-Tetri BA, Rinella M, Sanyal AJ. Mechanisms of NAFLD development and therapeutic strategies. Nat Med. 2018;24(7):908-922.

21. Noureddin M, Rinella ME. Nonalcoholic fatty liver disease, diabetes, obesity, and hepatocellular carcinoma. Clin Liver Dis. 2015;19(2): 361-379.
22. Targher G, Byrne CD, Lonardo A, Zoppini G, Barbui C. Non-alcoholic fatty liver disease and risk of incident cardiovascular disease: A metaanalysis. J Hepatol. 2016;65(3):589-600.

23. Hagström H, Nasr P, Ekstedt M, et al. Fibrosis stage but not NASH predicts mortality and time to development of severe liver disease in biopsy-proven NAFLD. J Hepatol. 2017;67(6):1265-1273.

24. Min YW, Kim Y, Gwak GY, et al. Non-alcoholic fatty liver disease and the development of reflux esophagitis: A cohort study. J Gastroenterol Hepatol. 2018;33(5):1053-1058. doi:10.1111/jgh.14042

25. Jia L, Haiyan L, Chengqi Z, et al. Non-alcoholic fatty liver disease associated with gallstones in females rather than males: A longitudinal cohort study in Chinese urban population. BMC Gastroenterol. 2014; 14:213.

26. Sahin A, Tunc N, Demirel U, Poyrazoglu OK, Yalniz M, Bahcecioglu IH. Relationship between diverticulosis and nonalcoholic fatty liver disease in elderly patients. J Int Med Res. 2018;46(4):1545-1554. 